\title{
No Quantum Ergodicity for Star Graphs
}

\author{
G. Berkolaiko ${ }^{1}$ \\ B. Winn ${ }^{2}$ \\ ${ }^{1}$ Department of Mathematics, University of Strathclyde, Glasgow G1 1XH, UK. \\ ${ }^{2}$ School of Mathematics, University of Bristol, Bristol BS8 1TW, UK.
}

$25^{\text {th }}$ July, 2003

\begin{abstract}
We investigate statistical properties of the eigenfunctions of the Schrödinger operator on families of star graphs with incommensurate bond lengths. We show that these eigenfunctions are not quantum ergodic in the limit as the number of bonds tends to infinity by finding an observable for which the quantum matrix elements do not converge to the classical average. We further show that for a given fixed graph there are subsequences of eigenfunctions which localise on pairs of bonds. We describe how to construct such subsequences explicitly. These constructions are analogous to scars on short unstable periodic orbits.
\end{abstract}

\section{Introduction}

Let $\psi_{n}$ denote the wave-function corresponding to the $n^{\text {th }}$ energy level of a quantum system that has a Hamiltonian dynamical system as its classical limit. We are interested in these wave-functions in the $n \rightarrow \infty$ limit, which corresponds to the semi-classical regime. Numerical and some theoretical evidence supports the hypothesis that their behaviour in this limit is determined by general properties of the underlying Hamiltonian such as, for example, time-reversibility, integrability and statistical properties of the flow (ergodicity, mixing, etc.). A deeper understanding of this is one of the goals of current research in quantum chaology.

When the classical Hamiltonian generates chaotic motion, the semi-classical eigenfunction hypothesis asserts that the wave-functions should equidistribute over the appropriate energy shell [Be1, $\mathrm{V}]$. A physical explanation for this is that in the semi-classical limit the quantum system should mimic the behaviour of the classical system; if the classical motion is chaotic, then a typical trajectory ergodically explores the surface of constant energy in phase space. Another interpretation is that eigenstates are invariant under time evolution, so it is natural to associate them in the semi-classical limit with classical invariant sets. One such invariant set is the energy shell itself.

The Schnirelman theorem [S, CdV, Z, GL] states that for systems in which the Hamiltonian flow is ergodic the sequence of measures induced by $\psi_{n}$ converges to Liouville measure in the limit as $n \rightarrow \infty$ along a subsequence of density one. This behaviour has been termed "quantum ergodicity". Quantum ergodicity implies a weak version of the semi-classical eigenfunction hypothesis [BSS]. 
It is possible that quantum ergodic systems have subsequences of states for which the corresponding measures do not converge to Liouville measure (of course such subsequences have density zero). These subsequences, if they exist, are expected to be associated with other classical invariant sets, such as periodic orbits. The case where the limit of an exceptional subseqence is a singular measure supported on one-or-more isolated, unstable periodic orbits of the classical system is called "scarring".

Scarred eigenstates were observed numerically by Heller $[\mathrm{H}]$, who proposed the first theoretical explanation for their existence, based on the semi-classical evolution of a wavepacket centred on a periodic orbit under linearised dynamics. Another important development was an understanding of the contribution to wave-functions from all periodic orbits [Bo, Be2] resulting in formulæ related to the semiclassical trace formula for the density of states. Later, the theory was extended to include non-linear effects $[\mathrm{KH}]$ and, more recently, situations where the orbit in question undergoes a bifurcation [KP. A review of related works was given in [K1. All of the above mentioned theories relate to scar effects in averages over a semiclassically increasing number of states. This may be thought of as a weakened form of scarring, because it is not clear that any one state in the averaging range causes the scar; the scars may be a collective effect. It is a much harder problem to show that a particular sequence of individual states is scarred. Currently, the only systems known rigorously to support scarring in this strong form are the cat maps [FNdB] which have non-generic spectral statistics caused by number-theoretical symmetries $\mathrm{Ke}$.

For quantum graphs KS1] the wave-functions are the eigenfunctions of the (continuous) Laplace operator on the bonds with matching conditions at the vertices chosen to make the problem self-adjoint. There is evidence to suggest that the spectral statistics of large quantum graphs coincide with those of generic quantised, classically chaotic, systems KS2, BSW1, BSW2, B] subject to mild conditions on the connectivity [T. Although the classical dynamics on a graph are not Hamiltonian, they are ergodic and it might be expected that an analogue of the Schnirelman theorem should hold.

Graphs are a rich source of problems in quantum chaology and related fields. Recent works have considered: scattering problems [KS3, TM, KS4, the spacing distribution of eigenvalues [BG], nodal domain statistics [GSW], the Dirac operator on graphs [BH1, BH2, Brownian motion on graphs [CDM] D] and the important question of how to construct families of graphs with increasing numbers of bonds PTZ.

Recently, authors have begun to investigate the wave-functions of quantum graphs. Kaplan [K2 studied eigenfunction statistics for ring-graphs using a combination of numerical techniques and analytical calculations of the short-time semiclassical behaviour of a wave-packet close to a 1-bond periodic orbit. The inverse participation ratio (a measure of localisation in a given state) was found to be well-described by this contribution, and shows deviation from the ergodically expected behaviour. Similar deviations were noticed for lattice-graphs. Remarkably, Schanz and Kottos [SK] observed that it would be impossible for the shortest orbits that are responsible for this enhanced localisation to support strong scarring. They wrote down an explicit criterion which must be satisfied by the energy of any strongly scarred state, and deduced asymptotics of the probability distribution of scarring strengths. In [KMW] a study was made of the eigenfunctions of a family of graphs known as star graphs (the name being derived from the connectivity of graphs in the family). The value distribution for the amplitude of eigenfunctions on a single bond of the graph, subject to an appropriate normalisation, was rigorously calculated 
in the limit as the number of bonds tends to infinity. In fact the normalisation implies that star graphs with a fixed, finite number of bonds are not quantum ergodic. However, this result leaves open the question of whether star graphs are quantum ergodic in the limit as the number of bonds tends to infinity. This is because one bond represents a vanishingly small fraction of a graph when the number of bonds becomes infinite, whereas quantum ergodicity is concerned with structures on macroscopic (classical) scales.

The results we present here extend the work in [KMW] on star graphs. We review the definition of a quantum star graph in section 2 below. We show that (see the following subsection for precise statements) quantum star graphs are not quantum ergodic in the limit as the number of bonds tends to infinity. We also show that for any given star graph there exist exceptional subsequences of eigenfunctions that become localised on pairs of bonds as $n \rightarrow \infty$. Orbits on a graph are simply itineraries of bonds, so this localisation is analogous to strong scarring on short period-2 orbits. Such orbits are unstable in the sense that there is an exponentially small probability of remaining on a given orbit. Our explicit construction supports the observation of Schanz and Kottos [SK] that star graphs support a large number of states scarred in such a way.

The spectral statistics of star graphs are different to those associated with the more general graphs described above $[\mathrm{BK}]$. The fact that quantum star graphs are not quantum ergodic does not contradict the possibility of a quantum ergodicity theorem for graphs with general connectivity. It is known that the spectral statistics of quantum star graphs are the same as those associated with the family of Šeba billiards [Se, BBK], so-called "intermediate statistics". There is evidence to suggest that the results we present on scarring can also be extended to Šeba billiards BKW].

\subsection{Main results}

To investigate quantum ergodicity for large star graphs, we consider an observable that picks out a positive proportion of the graph. We consider a graph with $\alpha v$ bonds, where $\alpha, v \in \mathbb{N}$, and the observable $\mathbf{B}=\left(B_{i}(x)\right)_{i=1}^{\alpha v}$ defined by

$$
B_{i}:= \begin{cases}1 & \text { for } i=1, \ldots, v \\ 0 & \text { for } i=v+1, \ldots, \alpha v .\end{cases}
$$

B may be thought of as the indicator function of the first $v$ bonds. The classical average of $\mathbf{B}$ is approximately $1 / \alpha$. We shall consider the limit $v \rightarrow \infty$.

Wave-functions on graphs have a component on each bond, so we shall use the notation

$$
\psi^{(n)}:=\left(\psi_{i}^{(n)}\right)_{i=1}^{\alpha v}
$$

for the $n^{\text {th }}$ eigenstate. The inner product $\langle\cdot \mid \cdot\rangle$ is defined in (2.8) below.

Each bond of the graph has a length, and the vector of bond lengths will be denoted $\mathbf{L}:=\left(L_{i}\right)_{i=1}^{\alpha v}$.

Theorem 1.1. For each $v$ let the components of $\mathbf{L}$ be linearly independent over $\mathbb{Q}$. Then there exists a probability density $p_{v}(\eta)$ such that for any continuous function $h$,

$$
\lim _{N \rightarrow \infty} \frac{1}{N} \sum_{n=1}^{N} h\left(\left\langle\boldsymbol{\psi}^{(n)}|\mathbf{B}| \boldsymbol{\psi}^{(n)}\right\rangle\right)=\int_{-\infty}^{\infty} h(\eta) p_{v}(\eta) \mathrm{d} \eta .
$$

The density $p_{v}(\eta)$ is supported on the interval $[0,1]$. 
Theorem 1.2. For each $v$ let the bond lengths $L_{j}, j=1, \ldots, \alpha v$ lie in the range $[\bar{L}, \bar{L}+\Delta L]$ and be linearly independent over $\mathbb{Q}$. If $v \Delta L \rightarrow 0$ as $v \rightarrow \infty$ then there exists a probability distribution function $F(R)$ such that for any $R \in(0,1)$,

$$
\lim _{v \rightarrow \infty} \int_{-\infty}^{R} p_{v}(\eta) \mathrm{d} \eta=F(R)
$$

where

$$
F(R)=\frac{1}{2}-\left.\frac{1}{\pi \alpha} \mathfrak{R e} \int_{-\infty}^{\infty} P_{\eta}(\xi)\left(\arg \left(\tau_{\eta}(\xi)\right)-\mathrm{i} \log \left|\tau_{\eta}(\xi)\right|\right) \mathrm{d} \xi\right|_{\eta=1 / R-1}
$$

and

$$
\begin{aligned}
P_{\eta}(\xi)= & \frac{1}{\sqrt{\pi \eta}} \exp \left(\frac{-\mathrm{i} \pi}{4}+\frac{\mathrm{i} \xi^{2}}{4 \eta}\right)+\frac{(\alpha-1)}{\sqrt{\pi}} \exp \left(\frac{\mathrm{i} \pi}{4}-\frac{\mathrm{i} \xi^{2}}{4}\right) \\
\tau_{\eta}(\xi)= & \frac{2}{\sqrt{\pi}} \sqrt{\eta} \exp \left(\frac{\mathrm{i} \pi}{4}+\frac{\mathrm{i} \xi^{2}}{4 \eta}\right)+\xi \operatorname{erf}\left(\frac{\mathrm{e}^{-\mathrm{i} \pi / 4} \xi}{2 \sqrt{\eta}}\right) \\
& +\frac{2(\alpha-1)}{\sqrt{\pi}} \exp \left(-\frac{\mathrm{i} \pi}{4}-\frac{\mathrm{i} \xi^{2}}{4}\right)+\xi(\alpha-1) \operatorname{erf}\left(\frac{\mathrm{e}^{\mathrm{i} \pi / 4} \xi}{2}\right)
\end{aligned}
$$

The function $F(R)$ is plotted in figure 1.

Remark 1.3. If star graphs satisfied quantum ergodicity, then $F(R)$ would be the stepfunction

$$
F(R)= \begin{cases}1, & \text { for } R>1 / \alpha \\ 0, & \text { for } R<1 / \alpha\end{cases}
$$

for this observable.

In figure 1 we compare the numerical data for the value distribution of $\left\langle\boldsymbol{\psi}^{(n)}|\mathbf{B}| \boldsymbol{\psi}^{(n)}\right\rangle$ for a star graph with 90 bonds with the $v \rightarrow \infty$ analytical prediction $F(R)$. The difference between the actual distribution $F(R)$ and that which would be expected if the graph were quantum ergodic (remark [1.3) is clear. Figure 2 shows the difference between numerical data and $F(R)$ for increasing values of $v$.

We we also show that for graphs with fixed number of bonds, there are subsequences of eigenfunctions that localise on two bonds.

Theorem 1.4. Let the elements of $\mathbf{L}$ be linearly independent over $\mathbb{Q}$. Given any distinct two bonds, indexed by $i_{1}$ and $i_{2}$, of a $v$-bond star graph, there exists a subsequence $\left(k_{n_{r}}\right) \subseteq$ $\left(k_{n}\right)$ such that for any $\mathbf{f}=\left(f_{i}\right)_{i=1}^{v}$ smooth in each component,

$$
\lim _{r \rightarrow \infty}\left\langle\boldsymbol{\psi}^{\left(n_{r}\right)}|\mathbf{f}| \boldsymbol{\psi}^{\left(n_{r}\right)}\right\rangle=\frac{1}{L_{i_{1}}+L_{i_{2}}}\left(\int_{0}^{L_{i_{1}}} f_{i_{1}}(x) \mathrm{d} x+\int_{0}^{L_{i_{2}}} f_{i_{2}}(x) \mathrm{d} x\right) .
$$




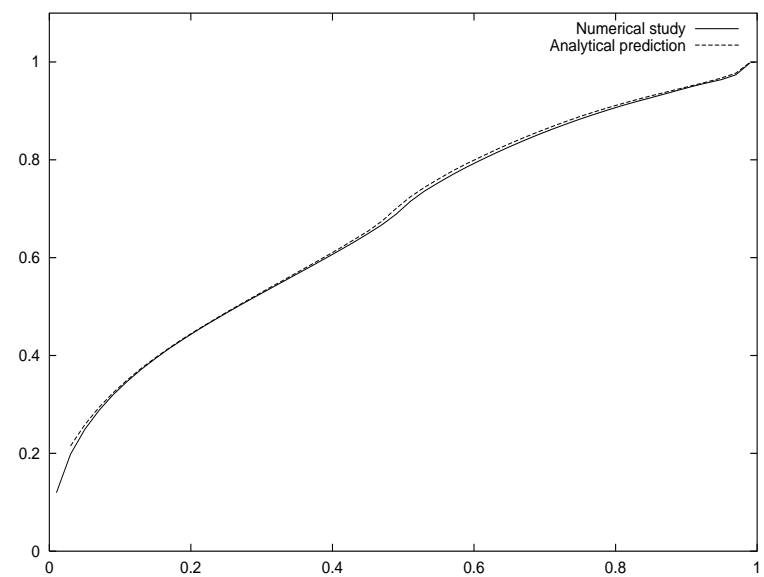

Figure 1: Comparing numerical data with the analytical prediction, $F(R)$. For this plot $\alpha=3$ and in the numerical study, $v=30$.

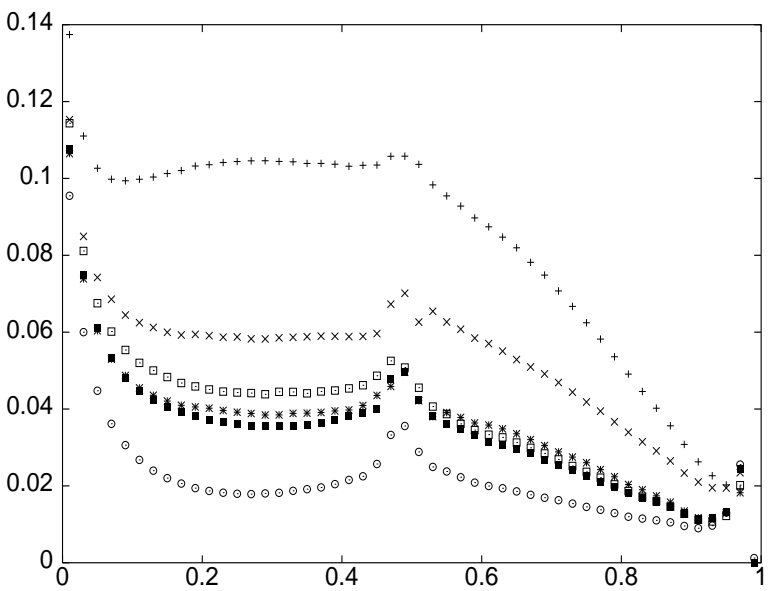

Figure 2: Convergence to $F(R)$ for $v=5(+), 10(\times), 15(*), 20(\bullet), 25(\mathbf{\square}), 30(\odot)$.

\section{Quantum Star Graphs}

A star graph ${ }^{1}$ is a metric graph with $b$ vertices all connected only to one central vertex. Thus there are $b+1$ vertices and $b$ bonds (figure $\mathbf{3}$ ). We shall denote by $\mathbf{L} \in \mathbb{R}^{b}$ the vector of bond lengths.

We define the quantum star graph in the following way. Let $\mathcal{H}$ denote the real Hilbert space

$$
\mathcal{H}:=L^{2}\left(\left[0, L_{1}\right]\right) \times \cdots \times L^{2}\left(\left[0, L_{b}\right]\right)
$$

with inner product

$$
\langle\mathbf{f} \mid \mathbf{g}\rangle:=\sum_{j=1}^{b} \int_{0}^{L_{j}} f_{j}(x) g_{j}(x) \mathrm{d} x .
$$

\footnotetext{
${ }^{1}$ sometimes referred to as a Hydra graph
} 


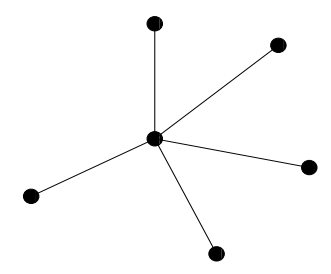

Figure 3: A star graph with 5 bonds

Elements of $\mathcal{H}$ are denoted $\mathbf{f}=\left(f_{1}, \ldots, f_{b}\right)$. Let $\mathcal{F} \subseteq \mathcal{H}$ be the subset of functions $\mathbf{f}$ which are twice-differentiable in each component and satisfy the conditions

$$
\begin{aligned}
f_{j}(0)=f_{i}(0) & =: f_{0}, \quad j, i=1, \ldots, b \\
\sum_{j=1}^{b} f_{j}^{\prime}(0) & =\frac{1}{\lambda} f_{0} \\
f_{j}^{\prime}\left(L_{j}\right) & =0, \quad j=1, \ldots, b .
\end{aligned}
$$

The parameter $\lambda$ may be varied to give different boundary conditions at the central vertex of the graph. Henceforth we shall concentrate on the case $1 / \lambda=0$, the so-called Neumann condition. The Laplace operator $\triangle$ on $\mathcal{F}$ is defined by

$$
\triangle \mathbf{f}:=\left(\frac{\mathrm{d}^{2} f_{1}}{\mathrm{~d} x^{2}}, \ldots, \frac{\mathrm{d}^{2} f_{b}}{\mathrm{~d} x^{2}}\right) .
$$

$\triangle$ defined on $\mathcal{F}$ is self-adjoint. Since the space on which the functions in $\mathcal{F}$ are defined is compact, the operator $\triangle$ has a discrete spectrum of eigenvalues ([DS], Section XIII.4). i.e. the equation

$$
-\triangle \boldsymbol{\psi}=k^{2} \boldsymbol{\psi}
$$

has non-trivial solutions for $k=k_{1}, k_{2}, \ldots$ Such $\boldsymbol{\psi}$ are the wave-functions [KS1, KS2]. We shall use the notation that $\boldsymbol{\psi}^{(n)}:=\left(\psi_{i}^{(n)}(x)\right)_{i=1}^{b}$ is the wave-function corresponding to $k=k_{n}$.

Solving (2.13) with boundary conditions (2.9) - 2.11), we find that the component of the $n^{\text {th }}$ normalised eigenfunction of the Laplace operator on the $i^{\text {th }}$ bond of a star graph is

$$
\psi_{i}^{(n)}(x)=A_{i}^{(n)} \cos k_{n}\left(x-L_{i}\right)
$$

where the amplitude is given by

$$
A_{i}^{(n)}=\left(\frac{2 \sec ^{2} k_{n} L_{i}}{\sum_{j=1}^{b} L_{j} \sec ^{2} k_{n} L_{j}}\right)^{1 / 2}
$$

and $k_{n}$ is the $n^{\text {th }}$ positive solution to

$$
Z(k, \mathbf{L}):=\sum_{j=1}^{b} \tan k L_{j}=0 .
$$

In sections 3 [ 15 it will be convenient to take $b=\alpha v$, where $\alpha \in \mathbb{N}$ is fixed. This is so that we can easily describe a fraction of the total number of bonds as the number of bonds becomes large $(v \rightarrow \infty)$. In section [6] we shall take $\alpha=1$ for notational convenience, since there we will only be concerned with fixed graphs. 


\section{Distribution of the observable B}

In this section we prove the existence of a limit distribution for the diagonal matrix elements of $\mathbf{B}$ on star graphs with a fixed number, $\alpha v$ of bonds.

Lemma 3.1. Consider a star graph with $\alpha v$ bonds with fixed lengths given by the vector L. Then for $\mathbf{B}$ defined by (1.1),

$$
\left\langle\boldsymbol{\psi}^{(n)}|\mathbf{B}| \boldsymbol{\psi}^{(n)}\right\rangle=\frac{\sum_{i=1}^{v} L_{i} \sec ^{2} k_{n} L_{i}}{\sum_{j=1}^{\alpha v} L_{j} \sec ^{2} k_{n} L_{j}}+\mathrm{O}\left(\frac{1}{k_{n}}\right)
$$

where the error estimate is uniform in $v$ and $L_{i} \geqslant L_{\min }>0$ for each $i$.

Proof. We recall that

$$
\left\langle\boldsymbol{\psi}^{(n)}|\mathbf{B}| \boldsymbol{\psi}^{(n)}\right\rangle=\sum_{j=1}^{\alpha v} \int_{0}^{L_{j}}\left|\psi_{j}^{(n)}(x)\right|^{2} B_{j}(x) \mathrm{d} x .
$$

Integrating (2.14) gives, for $i=1, \ldots, v$,

$$
\int_{0}^{L_{i}}\left|\psi_{i}^{(n)}(x)\right|^{2} B_{i}(x) \mathrm{d} x=\frac{1}{\sum_{j=1}^{\alpha v} L_{j} \sec ^{2} k_{n} L_{j}}\left(L_{i} \sec ^{2} k_{n} L_{i}+\frac{1}{k_{n}} \tan k_{n} L_{i}\right),
$$

and for $i \geqslant v+1$,

$$
\int_{0}^{L_{i}}\left|\psi_{i}^{(n)}(x)\right|^{2} B_{i}(x) \mathrm{d} x=0
$$

Thus

$$
\left\langle\boldsymbol{\psi}^{(n)}|\mathbf{B}| \boldsymbol{\psi}^{(n)}\right\rangle=\frac{\sum_{i=1}^{v} L_{i} \sec ^{2} k_{n} L_{i}}{\sum_{j=1}^{\alpha v} L_{j} \sec ^{2} k_{n} L_{j}}+\frac{E}{k_{n}}
$$

where

$$
E=\frac{\sum_{i=1}^{v} \tan k_{n} L_{i}}{\sum_{j=1}^{\alpha v} L_{j} \sec ^{2} k_{n} L_{j}}
$$

Let $L_{\min }:=\min _{j}\left\{L_{j}\right\}$. Then

$$
\begin{aligned}
|E| & \leqslant \frac{\sum_{i=1}^{v}\left|\tan k_{n} L_{i}\right|}{(\alpha-1) L_{\min } v+L_{\min } \sum_{j=1}^{v} \sec ^{2} k_{n} L_{j}} \\
& \leqslant\left(L_{\min }+\frac{(\alpha-1) v L_{\min }}{\sum_{i=1}^{v} \sec ^{2} k_{n} L_{j}}\right)^{-1}
\end{aligned}
$$

using the fact that $|\tan \theta| \leqslant \sec ^{2} \theta$ for any $\theta \in \mathbb{R}$. Hence $E=\mathrm{O}(1)$ as $n \rightarrow \infty$ uniformly in $v, L_{\min }>0$.

Proof of theorem [1.1. By lemma 3.1.

$$
h\left(\left\langle\boldsymbol{\psi}^{(n)}|\mathbf{B}| \boldsymbol{\psi}^{(n)}\right\rangle\right)=h\left(\frac{\sum_{i=1}^{v} L_{i} \sec ^{2} x_{i}}{\sum_{j=1}^{\alpha v} L_{j} \sec ^{2} x_{j}}\right)+E_{n}
$$

where $E_{n}=\mathrm{o}(1)$ as $n \rightarrow \infty$ since $h$ is uniformly continuous on $[0,1]$. Hence

$$
\frac{1}{N} \sum_{n=1}^{N} E_{n} \rightarrow 0 \quad \text { as } N \rightarrow \infty
$$


Therefore

$$
\lim _{N \rightarrow \infty} \frac{1}{N} \sum_{n=1}^{N} h\left(\left\langle\boldsymbol{\psi}^{(n)}|\mathbf{B}| \boldsymbol{\psi}^{(n)}\right\rangle\right)=\lim _{N \rightarrow \infty} \frac{1}{N} \sum_{n=1}^{N} h\left(\frac{\sum_{i=1}^{v} L_{i} \sec ^{2} k_{n} L_{i}}{\sum_{j=1}^{\alpha v} L_{j} \sec ^{2} k_{n} L_{j}}\right) .
$$

According to Barra and Gaspard, there is an absolutely continuous measure $\nu(\boldsymbol{\xi})$ such that for piecewise continuous functions, $f: \Sigma \rightarrow \mathbb{R}$,

$$
\lim _{N \rightarrow \infty} \frac{1}{N} \sum_{n=1}^{N} f\left(k_{n} \mathbf{L}\right)=\int_{\Sigma} f(\boldsymbol{\xi}) \mathrm{d} \nu(\boldsymbol{\xi})
$$

where $\Sigma$ is the surface embedded in the $\alpha v$ dimensional torus with side $\pi$, defined by

$$
\tan x_{1}+\cdots+\tan x_{\alpha v}=0 .
$$

$\xi$ is a set of $\alpha v-1$ coordinates which parameterise $\Sigma$. To avoid repetition, we refer the reader to $[\mathrm{BG}$, $\mathrm{KMW}$ ] for more detail about this result and its application to similar problems.

Let

$$
f(\mathbf{x})=h\left(\frac{\sum_{i=1}^{v} L_{i} \sec ^{2} x_{i}}{\sum_{j=1}^{\alpha v} L_{j} \sec ^{2} x_{j}}\right)
$$

we can define $p_{v}(\eta)$ by

$$
\int_{\Sigma} f(\boldsymbol{\xi}) \mathrm{d} \nu(\boldsymbol{\xi})=: \int_{-\infty}^{\infty} h(\eta) p_{v}(\eta) \mathrm{d} \eta
$$

Since $0 \leqslant\left\langle\boldsymbol{\psi}^{(n)}|\mathbf{B}| \boldsymbol{\psi}^{(n)}\right\rangle \leqslant 1$, it follows that $p_{v}(\eta)$ is supported on $[0,1]$.

\section{The large graph limit}

Let $\eta \in \mathbb{R}$ and define

$$
X_{\eta}(n):=\frac{1}{v^{2}} \sum_{j=v+1}^{\alpha v} L_{j} \sec ^{2} k_{n} L_{j}-\frac{\eta}{v^{2}} \sum_{i=1}^{v} L_{i} \sec ^{2} k_{n} L_{i}
$$

for $n=1,2, \ldots$ The key result of this section is the following.

Proposition 4.1. For each $v$, there exists a probability density function $f_{X_{\eta}, v}$ such that

$$
\lim _{N \rightarrow \infty} \frac{1}{N} \#\left\{n \in\{1, \ldots, N\}: X_{\eta}(n)<S\right\}=\int_{-\infty}^{S} f_{X_{\eta}, v}(\sigma) \mathrm{d} \sigma .
$$

Furthermore, for each $S \in \mathbb{R}$,

$$
\int_{-\infty}^{S} f_{X_{\eta}, v}(\sigma) \mathrm{d} \sigma \rightarrow \int_{-\infty}^{S} f_{X_{\eta}}(\sigma) \mathrm{d} \sigma
$$

as $v \rightarrow \infty$, provided that $v \Delta L \rightarrow 0$ in this limit, where

$$
f_{X_{\eta}}(\sigma)=\frac{-1}{4 \alpha \sqrt{\pi}} \mathfrak{R e} \int_{-\infty}^{\infty} P_{\eta}(\xi) \frac{\mathrm{e}^{3 \mathrm{i} \pi / 4} \tau_{\eta}(\xi)}{(-\sigma)^{3 / 2}} w\left(\frac{\mathrm{e}^{3 \mathrm{i} \pi / 4} \tau_{\eta}(\xi)}{2 \sqrt{-\sigma}}\right) \mathrm{d} \xi .
$$

The functions $P_{\eta}$ and $\tau_{\eta}$ are defined by (4.43) and (4.44) below, and $w(z):=\mathrm{e}^{-z^{2}} \operatorname{erfc}(-\mathrm{i} z)$. 
Proof. The existence of the $N \rightarrow \infty$ limiting density, $f_{X_{\eta}, v}$ is a consequence of the result of Barra and Gaspard [BG]. The proof is entirely analogous to the proof of theorem [1.1.

We turn our attention to the $v \rightarrow \infty$ limit of this density. With $n$ a random variable uniformly distributed on the set $\{1, \ldots, N\}$ for some $N \in \mathbb{N}$, the characteristic function for this random variable $X_{\eta}(n)$ is

$$
e_{v, N}(\beta):=\mathbb{E}\left(\mathrm{e}^{\mathrm{i} \beta X_{\eta}}\right)=\frac{1}{N} \sum_{n=1}^{N} f\left(k_{n} \mathbf{L}\right)+\mathrm{O}(v \Delta L)
$$

where $f:[0, \pi]^{v} \rightarrow \mathbb{C}$ is defined to be

$$
f(\mathbf{x}):=\exp \left(\frac{\mathrm{i} \beta}{v^{2}}\left(\sum_{j=v+1}^{\alpha v} \sec ^{2} x_{j}-\eta \sum_{i=1}^{v} \sec ^{2} x_{i}\right)\right) .
$$

Following the argument of [KMW] we can write

$$
\begin{aligned}
\lim _{N \rightarrow \infty} & \frac{1}{N} \sum_{n=1}^{N} f\left(k_{n} \mathbf{L}\right) \\
& =\frac{1}{2 \alpha v^{2}} \frac{1}{\pi^{\alpha v}} \int_{-\infty}^{\infty} \int_{0}^{\pi} \cdots \int_{0}^{\pi}\left(\sum_{j=1}^{\alpha v} \sec ^{2} x_{j}\right) f(\mathbf{x}) \exp \left(\frac{\mathrm{i} \zeta}{v} \sum_{j=1}^{\alpha v} \tan x_{j}\right) \mathrm{d} \alpha v \mathbf{x} \mathrm{d} \zeta .
\end{aligned}
$$

Denoting this limit for each fixed $v, \beta$ by $e_{v}(\beta)$, we can write

$$
e_{v}(\beta)=\frac{1}{2 \alpha v} \int_{-\infty}^{\infty} I_{1} I_{2}^{v-1} I_{3}^{\alpha v-v}+(\alpha-1) I_{4} I_{2}^{v} I_{3}^{\alpha v-v-1} \mathrm{~d} \zeta
$$

where the integrals $I_{1}, \ldots, I_{4}$ are:-

$$
\begin{aligned}
& I_{1}:=\frac{1}{\pi} \int_{0}^{\pi} \sec ^{2} x \exp \left(\frac{\mathrm{i} \zeta}{v} \tan x-\frac{\mathrm{i} \beta \eta}{v^{2}} \sec ^{2} x\right) \mathrm{d} x, \\
& I_{2}:=\frac{1}{\pi} \int_{0}^{\pi} \exp \left(\frac{\mathrm{i} \zeta}{v} \tan x-\frac{\mathrm{i} \beta \eta}{v^{2}} \sec ^{2} x\right) \mathrm{d} x \\
& I_{3}:=\frac{1}{\pi} \int_{0}^{\pi} \exp \left(\frac{\mathrm{i} \zeta}{v} \tan x+\frac{\mathrm{i} \beta}{v^{2}} \sec ^{2} x\right) \mathrm{d} x \\
& I_{4}:=\frac{1}{\pi} \int_{0}^{\pi} \sec ^{2} x \exp \left(\frac{\mathrm{i} \zeta}{v} \tan x+\frac{\mathrm{i} \beta}{v^{2}} \sec ^{2} x\right) \mathrm{d} x
\end{aligned}
$$

An integral similar to (4.32) was tackled in [KMW]. We quote here the relevant results, mutatis mutandis. Asymptotic analysis of the integrals in (4.33 4.36) gives

$$
I_{1}=\frac{v}{\sqrt{\pi \beta \eta}} \exp \left(-\frac{\mathrm{i} \pi}{4}+\frac{\mathrm{i} \zeta^{2}}{4 \beta \eta}\right)+\mathrm{O}(1) \quad \text { as } v \rightarrow \infty
$$

and

$$
I_{4}=\frac{v}{\sqrt{\pi \beta}} \exp \left(\frac{\mathrm{i} \pi}{4}-\frac{\mathrm{i} \zeta^{2}}{4 \beta}\right)+\mathrm{O}(1) \quad \text { as } v \rightarrow \infty .
$$


For $I_{2}$ and $I_{3}$ we consider separately the cases $-\sqrt{v}<\zeta<\sqrt{v}$ and $|\zeta|>\sqrt{v}$. For $-\sqrt{v}<\zeta<\sqrt{v}$

$$
I_{2}^{v}=\exp \left(-\frac{2}{\sqrt{\pi}} \sqrt{\beta \eta} \exp \left(\frac{\mathrm{i} \pi}{4}+\frac{\mathrm{i} \zeta^{2}}{4 \beta \eta}\right)-\zeta \operatorname{erf}\left(\frac{\mathrm{e}^{-\mathrm{i} \pi / 4} \zeta}{2 \sqrt{\beta \eta}}\right)\right)\left(1+\mathrm{O}\left(\frac{1+\zeta^{2}}{v}\right)\right)
$$

and

$$
\begin{aligned}
I_{3}^{(\alpha-1) v}=\exp \left(-\frac{2(\alpha-1)}{\sqrt{\pi}} \sqrt{\beta} \exp \left(-\frac{\mathrm{i} \pi}{4}-\frac{\mathrm{i} \zeta^{2}}{4 \beta}\right)-\zeta(\alpha-1) \operatorname{erf}\right. & \left.\left(\frac{\mathrm{e}^{\mathrm{i} \pi / 4} \zeta}{2 \sqrt{\beta}}\right)\right) \\
& \times\left(1+\mathrm{O}\left(\frac{1+\zeta^{2}}{v}\right)\right),
\end{aligned}
$$

both error estimates are as $v \rightarrow \infty$. For $|\zeta|>\sqrt{v}$

$$
\left|I_{2}\right| \leqslant \frac{\sqrt{\beta \eta}}{v \pi}\left(\frac{\beta \eta}{v^{2}}+\frac{\zeta^{2}}{\beta \eta}\right)^{-1}+\mathrm{O}\left(\zeta^{-3}\right)
$$

as $\zeta \rightarrow \infty$, and

$$
\left|I_{3}\right| \leqslant \frac{\sqrt{\beta}}{v \pi}\left(\frac{\beta}{v^{2}}+\frac{\zeta^{2}}{\beta}\right)^{-1}+\mathrm{O}\left(\zeta^{-3}\right)
$$

Using these estimates, we can find an expression for the limit of $e_{v}(\beta)$ as $v \rightarrow \infty$,

$$
\begin{aligned}
e(\beta):=\lim _{v \rightarrow \infty} e_{v}(\beta) & =\frac{1}{2 \alpha} \int_{-\infty}^{\infty} \frac{1}{\sqrt{\beta}} P_{\eta}\left(\frac{\zeta}{\sqrt{\beta}}\right) \exp \left(-\sqrt{\beta} \tau_{\eta}\left(\frac{\zeta}{\sqrt{\beta}}\right)\right) \mathrm{d} \zeta \\
& =\int_{-\infty}^{\infty} P_{\eta}(\xi) \exp \left(-\sqrt{\beta} \tau_{\eta}(\xi)\right) \mathrm{d} \xi
\end{aligned}
$$

For ease of notation, we have introduced

$$
P_{\eta}(\xi):=\frac{1}{\sqrt{\pi \eta}} \exp \left(\frac{-\mathrm{i} \pi}{4}+\frac{\mathrm{i} \xi^{2}}{4 \eta}\right)+\frac{(\alpha-1)}{\sqrt{\pi}} \exp \left(\frac{\mathrm{i} \pi}{4}-\frac{\mathrm{i} \xi^{2}}{4}\right)
$$

and

$$
\begin{aligned}
\tau_{\eta}(\xi):= & \frac{2}{\sqrt{\pi}} \sqrt{\eta} \exp \left(\frac{\mathrm{i} \pi}{4}+\frac{\mathrm{i} \xi^{2}}{4 \eta}\right)+\xi \operatorname{erf}\left(\frac{\mathrm{e}^{-\mathrm{i} \pi / 4} \xi}{2 \sqrt{\eta}}\right) \\
& +\frac{2(\alpha-1)}{\sqrt{\pi}} \exp \left(-\frac{\mathrm{i} \pi}{4}-\frac{\mathrm{i} \xi^{2}}{4}\right)+\xi(\alpha-1) \operatorname{erf}\left(\frac{\mathrm{e}^{\mathrm{i} \pi / 4} \xi}{2}\right) .
\end{aligned}
$$

In the above, wherever $\sqrt{\beta}$ occurs for $\beta<0$, this should be understood to mean $\pm \mathrm{i} \sqrt{-\beta}$ where the sign is taken in such a way that

$$
e(-\beta)=\overline{e(\beta)}
$$

the usual condition for the characteristic function of a probability density. This can always be done. We also note that $e(0)=1$ which is consistent with $e(\beta)$ being the characteristic function of a probability distribution. $e(\beta)$ is continuous at $\beta=0$ since the defining 
integral is uniformly convergent in $\beta$ (see lemma A.3 below). Thus the limiting density, $f_{X_{\eta}}$ exists and is given by

$$
f_{X_{\eta}}(\sigma)=\frac{1}{2 \pi} \int_{-\infty}^{\infty} \frac{1}{2 \alpha} \int_{-\infty}^{\infty} P_{\eta}(\xi) \exp \left(-\sqrt{\beta} \tau_{\eta}(\xi)-\mathrm{i} \sigma \beta\right) \mathrm{d} \xi \mathrm{d} \beta
$$

where we have made the substitution $\xi=\zeta / \sqrt{\beta}$. We here switch the order of integration. This is a non-trivial operation since both integrals are improper. However in this case we can rigorously justify the manoeuvre. Justification is provided in appendix A proposition A.8.

$$
\begin{aligned}
f_{X_{\eta}}(\sigma) & =\frac{1}{2 \pi \alpha} \mathfrak{R e} \int_{-\infty}^{\infty} P_{\eta}(\xi) \int_{0}^{\infty} \exp \left(-\sqrt{\beta} \tau_{\eta}(\xi)-\mathrm{i} \sigma \beta\right) \mathrm{d} \beta \mathrm{d} \xi \\
& =\frac{1}{2 \pi \alpha} \mathfrak{R e} \int_{-\infty}^{\infty} P_{\eta}(\xi)\left(\frac{1}{\mathrm{i} \sigma}-\frac{\sqrt{\pi}}{2} \frac{\mathrm{e}^{3 \mathrm{i} \pi / 4} \tau_{\eta}(\xi)}{(-\sigma)^{3 / 2}} w\left(\frac{\mathrm{e}^{3 \mathrm{i} \pi / 4} \tau_{\eta}(\xi)}{2 \sqrt{-\sigma}}\right)\right) \mathrm{d} \xi
\end{aligned}
$$

To evaluate the final integration we have used the following result (a variant of formula 3.462.5 in [GR]),

$$
\int_{0}^{\infty} \exp (-a x-b \sqrt{x}) \mathrm{d} x=\frac{1}{a}-\frac{\sqrt{\pi}}{2} \frac{b}{a^{3 / 2}} w\left(\frac{\mathrm{i} b}{2 a^{1 / 2}}\right) .
$$

To conclude we observe that since

$$
\int_{-\infty}^{\infty} P_{\eta}(\xi) \mathrm{d} \xi=2 \alpha \in \mathbb{R}
$$

the first term in (4.46) vanishes as it has zero real part.

Some properties of $w(z)$ are discussed in [AS] chapter 7. We shall use the following

Lemma 4.2. The function $w(z)$ has the asymptotic expansion

$$
w(z) \sim \frac{\mathrm{i}}{\sqrt{\pi}} \sum_{m=0}^{\infty} \frac{(2 m) !}{4^{m} m ! z^{2 m+1}}
$$

as $z \rightarrow \infty$, valid for $\frac{-\pi}{4}<\arg z<\frac{5 \pi}{4}$.

Proof. This follows from the asymptotic expansion of erfc,

$$
\sqrt{\pi} z \mathrm{e}^{z^{2}} \operatorname{erfc}(z) \sim \sum_{m=0}^{\infty} \frac{(2 m) !}{\left(4 z^{2}\right)^{m} m !},
$$

as $z \rightarrow \infty,|\arg z|<3 \pi / 4$, taken from [AS] (formula 7.1.23; see also [BlHa] exercise 3.11). The series for $w$ comes from making the substitution $z \mapsto-\mathrm{i} z$.

Since erfc and $w$ are analytic functions, they are bounded in the domain of validity of their asymptotic expansions quoted in lemma 4.2.

Proposition 4.3. Let $z \in \mathbb{C}$ and $-\pi / 4<\arg z<5 \pi / 4$. Then

$$
\int_{0}^{R} z w(z p) \mathrm{d} p=\frac{\sqrt{\pi}}{2}-\frac{\arg (z)}{\sqrt{\pi}}+\frac{\mathrm{i}}{\sqrt{\pi}} \log |z|+\mathrm{i} C_{R}+\mathrm{O}\left(\frac{1}{|z|^{2} R^{2}}\right)
$$

where $C_{R} \in \mathbb{R}$ is independent of $z$, but may depend on $R$. 


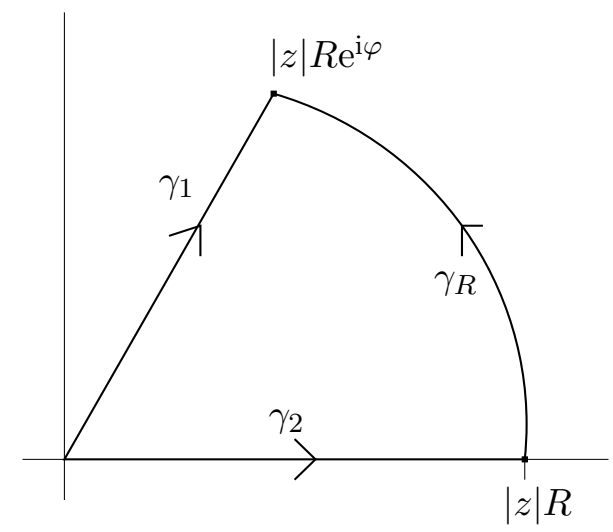

Figure 4: The contours $\gamma_{1}, \gamma_{2}$ and $\gamma_{R}$.

Proof. Write $z=|z| \mathrm{e}^{\mathrm{i} \varphi}$ where $\varphi=\arg z$. Then

$$
\int_{0}^{R} z w(z p) \mathrm{d} p=\int_{0}^{|z| R} \mathrm{e}^{\mathrm{i} \varphi} w\left(\mathrm{e}^{\mathrm{i} \varphi} p\right) \mathrm{d} p
$$

via $p \mapsto p /|z|$. Using Cauchy's theorem,

$$
\int_{0}^{|z| R} \mathrm{e}^{\mathrm{i} \varphi} w\left(\mathrm{e}^{\mathrm{i} \varphi} p\right) \mathrm{d} p=\int_{\gamma_{1}} w(t) \mathrm{d} t=\int_{\gamma_{2}} w(t) \mathrm{d} t+\int_{\gamma_{R}} w(t) \mathrm{d} t .
$$

The contours $\gamma_{1}, \gamma_{2}$ and $\gamma_{R}$ in the complex $t$-plane are illustrated in figure 4

On $\gamma_{2}$,

$$
\begin{aligned}
\int_{\gamma_{2}} w(t) \mathrm{d} t= & \int_{0}^{|z| R} w(x) \mathrm{d} x \\
= & \int_{0}^{1} w(x) \mathrm{d} x+\int_{1}^{|z| R}\left(w(x)-\frac{\mathrm{i}}{\sqrt{\pi} x}\right) \mathrm{d} x+\int_{1}^{|z| R} \frac{\mathrm{i}}{\sqrt{\pi} x} \mathrm{~d} x \\
= & \int_{0}^{1} w(x) \mathrm{d} x+\int_{1}^{\infty}\left(w(x)-\frac{\mathrm{i}}{\sqrt{\pi} x}\right) \mathrm{d} x \\
& -\int_{|z| R}^{\infty}\left(w(x)-\frac{\mathrm{i}}{\sqrt{\pi} x}\right) \mathrm{d} x+\int_{1}^{|z| R} \frac{\mathrm{i}}{\sqrt{\pi} x} \mathrm{~d} x \\
= & \int_{0}^{\infty} \mathrm{e}^{-x^{2}} \mathrm{~d} x+\mathrm{i} \times \text { const }-\int_{|z| R}^{\infty}\left(w(x)-\frac{\mathrm{i}}{\sqrt{\pi} x}\right) \mathrm{d} x+\int_{1}^{|z| R} \frac{\mathrm{i}}{\sqrt{\pi} x} \mathrm{~d} x,
\end{aligned}
$$

using $w(z)=\mathrm{e}^{-x^{2}}(1+\mathrm{i} \operatorname{erfi} z)$ to separate the real and imaginary contributions. The imaginary part goes into the constant $C_{R}$, the value of which is not important since we shall always be considering only the real part of resulting expressions. By the use of lemma 4.2 .

$$
\int_{\gamma_{2}} w(t) \mathrm{d} t=\frac{\sqrt{\pi}}{2}+\mathrm{i} \times \text { const }+\frac{\mathrm{i}}{\sqrt{\pi}}(\log R+\log |z|)+\mathrm{O}\left(\frac{1}{|z|^{2} R^{2}}\right)
$$


as $R \rightarrow \infty$ uniformly for $|z| \geqslant c$ for some $c$.

On $\gamma_{R}$,

$$
\begin{aligned}
\int_{\gamma_{R}} f(t) \mathrm{d} t & =\int_{0}^{\varphi} \mathrm{i}|z| R \mathrm{e}^{\mathrm{i} \theta} w\left(|z| R \mathrm{e}^{\mathrm{i} \theta}\right) \mathrm{d} \theta \\
& =\int_{0}^{\varphi} \mathrm{i}|z| R \mathrm{e}^{\mathrm{i} \theta}\left(\frac{-1}{\sqrt{\pi} \mathrm{i}|z| R \mathrm{e}^{\mathrm{i} \theta}}+\mathrm{O}\left(\frac{1}{|z|^{3} R^{3}}\right)\right) \quad \text { by lemma 4.2 } \\
& =\frac{-\varphi}{\sqrt{\pi}}+\mathrm{O}\left(\frac{1}{|z|^{2} R^{2}}\right) .
\end{aligned}
$$

Combining (4.52) and (4.53) gives (4.49).

The following lemma from probability theory will also be useful.

Lemma 4.4. Let $U, V$ be random variables, then

$$
\mathbb{P}\left(\frac{U}{V}<\eta\right)=\int_{-\infty}^{0} f_{X_{\eta}}(\sigma) \mathrm{d} \sigma
$$

where $f_{X_{\eta}}$ is the probability density function of the random variable $X_{\eta}:=U-\eta V$.

Proof. This follows immediately from the fact that

$$
\mathbb{P}\left(\frac{U}{V}<\eta\right)=\mathbb{P}(U-\eta V<0) .
$$

\section{Proof of theorem 1.2}

We first observe that

$$
\frac{\sum_{i=1}^{v} L_{i} \sec ^{2} k_{n} L_{i}}{\sum_{j=1}^{\alpha v} L_{j} \sec ^{2} k_{n} L_{j}}=\left(1+\frac{\sum_{j=v+1}^{\alpha v} L_{j} \sec ^{2} k_{n} L_{j}}{\sum_{1=1}^{v} L_{i} \sec ^{2} k_{n} L_{i}}\right)^{-1},
$$

so we can concentrate on finding the probability distribution, $\tilde{F}(\eta)$, of

$$
\frac{\sum_{j=v+1}^{\alpha v} L_{j} \sec ^{2} k_{n} L_{j}}{\sum_{1=1}^{v} L_{i} \sec ^{2} k_{n} L_{i}}
$$

as $v \rightarrow \infty$. This will be then related to the distribution in which we are interested by a simple transformation (see equation (5.57) below). In light of lemma 4.4, $\tilde{F}(\eta)$ is given by

$$
\tilde{F}(\eta)=\int_{-\infty}^{0} f_{X_{\eta}}(\sigma) \mathrm{d} \sigma
$$

with $f_{X_{\eta}}$ found in proposition 4.1] It is more instructive to take the range of integration from $-R^{2}$ to 0 , with a view to taking the limit $R \rightarrow \infty$ later,

$$
\int_{-R^{2}}^{0} f_{X_{\eta}}(\sigma) \mathrm{d} \sigma=\frac{-1}{\sqrt{\pi} \alpha} \mathfrak{R e} \int_{-\infty}^{\infty} \int_{-R^{2}}^{0} P_{\eta}(\xi) \frac{\mathrm{e}^{3 \pi \mathrm{i} / 4} \tau_{\eta}(\xi)}{4(-\sigma)^{3 / 2}} w\left(\frac{\mathrm{e}^{3 \pi \mathrm{i} / 4} \tau_{\eta}(\xi)}{\sqrt{-\sigma}}\right) \mathrm{d} \sigma \mathrm{d} \xi
$$


The $\xi$-integral is uniformly convergent by lemma B.1 so we have legitimately switched the order of integration. We can then write

$$
\begin{aligned}
\int_{-R^{2}}^{0} f_{X_{\eta}}(\sigma) \mathrm{d} \sigma= & \frac{-1}{\alpha \sqrt{\pi}} \mathfrak{R e} \int_{-\infty}^{\infty} \int_{-\infty}^{0} P_{\eta}(\xi) \frac{\mathrm{e}^{3 \pi \mathrm{i} / 4} \tau_{\eta}(\xi)}{4(-\sigma)^{3 / 2}} w\left(\frac{\mathrm{e}^{3 \pi \mathrm{i} / 4} \tau_{\eta}(\xi)}{\sqrt{-\sigma}}\right) \mathrm{d} \sigma \mathrm{d} \xi \\
& -\frac{-1}{\sqrt{\pi} \alpha} \mathfrak{R e} \int_{-\infty}^{\infty} \int_{-\infty}^{-R^{2}} P_{\eta}(\xi) \frac{\mathrm{e}^{3 \pi \mathrm{i} / 4} \tau_{\eta}(\xi)}{4(-\sigma)^{3 / 2}} w\left(\frac{\mathrm{e}^{3 \pi \mathrm{i} / 4} \tau_{\eta}(\xi)}{\sqrt{-\sigma}}\right) \mathrm{d} \sigma \mathrm{d} \xi
\end{aligned}
$$

The second term vanishes as $R \rightarrow \infty$, as shown in proposition B.2. Making the substitution $(-\sigma)^{-1 / 2}=2 p$, leads to

$$
\tilde{F}(\eta)=\frac{-1}{\sqrt{\pi} \alpha} \mathfrak{R e} \int_{-\infty}^{\infty} \int_{0}^{\infty} P_{\eta}(\xi) \mathrm{e}^{3 \pi \mathrm{i} / 4} \tau_{\eta}(\xi) w\left(\mathrm{e}^{3 \pi \mathrm{i} / 4} \tau_{\eta}(\xi) p\right) \mathrm{d} p \mathrm{~d} \xi
$$

We can apply proposition 4.3 with $z=\mathrm{e}^{3 \mathrm{i} \pi / 4} \tau_{\eta}(\xi)$. We can integrate $\xi$ out of the error term provided by this proposition since

$$
\int_{-\infty}^{\infty} \frac{1}{\tau_{\eta}(\xi)^{2}} \mathrm{~d} \xi<\infty
$$

We get, finally,

$$
\begin{aligned}
\tilde{F}(\eta) & =\frac{-1}{\alpha \sqrt{\pi}} \mathfrak{R e} \int_{-\infty}^{\infty} P_{\eta}(\xi)\left(\frac{\sqrt{\pi}}{2}-\frac{\arg \left(\mathrm{e}^{3 \mathrm{i} \pi / 4} \tau_{\eta}(\xi)\right)}{\sqrt{\pi}}+\mathrm{i} \frac{\log \left|\tau_{\eta}(\xi)\right|}{\sqrt{\pi}}\right) \mathrm{d} \xi \\
& =\frac{1}{2}+\frac{1}{\pi \alpha} \mathfrak{R e} \int_{-\infty}^{\infty} P_{\eta}(\xi)\left(\arg \left(\tau_{\eta}(\xi)\right)-\mathrm{i} \log \left|\tau_{\eta}(\xi)\right|\right) \mathrm{d} \xi
\end{aligned}
$$

This is related to $F(\eta)$ by

$$
F(\eta)=1-\tilde{F}\left(\frac{1}{\eta}-1\right)
$$

\section{$6 \quad$ Scarred states on finite star graphs}

We recall that theorem 1.4 is concerned with the quantity $\left\langle\boldsymbol{\psi}^{(n)}|\mathbf{f}| \boldsymbol{\psi}^{(n)}\right\rangle$ which can be written as

$$
\left\langle\boldsymbol{\psi}^{(n)}|\mathbf{f}| \boldsymbol{\psi}^{(n)}\right\rangle=\sum_{i=1}^{v} \int_{0}^{L_{i}}\left|\psi_{i}^{(n)}(x)\right|^{2} f_{i}(x) \mathrm{d} x .
$$

Now using (2.14) and the identity $\cos ^{2} \theta=1 / 2+(1 / 2) \cos 2 \theta$ yields

$$
\left\langle\boldsymbol{\psi}^{(n)}|\mathbf{f}| \boldsymbol{\psi}^{(n)}\right\rangle=\sum_{i=1}^{v} \frac{A_{i}^{(n)^{2}}}{2}\left(\int_{0}^{L_{i}} f_{i}(x) \mathrm{d} x+\int_{0}^{L_{i}} \cos 2 k_{n}\left(x-L_{i}\right) f_{i}(x) \mathrm{d} x\right) .
$$

Since we are interested in a subsequence $k_{n_{r}} \rightarrow \infty$, we may hope that the second integrals do not survive. Thus our prime concern are the prefactors

$$
A_{i}^{(n)^{2}}=\frac{2 \sec ^{2} k_{n} L_{i}}{\sum_{j=1}^{v} L_{j} \sec ^{2} k_{n} L_{j}}
$$


and to prove the theorem we need to find $k_{n}$ such that the prefactors are small for all $i$ other than (without loss of generality) 1 and 2 .

To do this we study the poles of the function $Z(k, \mathbf{L})$. Let

$$
p_{n, i}:=\frac{\pi}{L_{i}}\left(\frac{1}{2}+n\right) \text {. }
$$

Then $\left|\tan p L_{i}\right| \rightarrow \infty$ and $\sec p L_{i} \rightarrow \infty$ as $p \rightarrow p_{n, i}$.

Since the function $Z(k, \mathbf{L})$ is an increasing function of $k$, between any two poles there is a zero. We will use this important feature to "trap" zeros $k_{n_{r}}$ of $Z(k, \mathbf{L})$ between pairs of nearby poles $p_{n, 1}$ and $p_{m, 2}$, also requiring that all other poles are far away (see Fig. (5) . The implications will be that as $r \rightarrow \infty$,

$$
\sec ^{2} k_{n_{r}} L_{i} \gg \sec ^{2} k_{n_{r}} L_{j} \quad \text { with } i=1,2 \text { and } j>2,
$$

and

$$
\sec ^{2} k_{n_{r}} L_{1} \sim \sec ^{2} k_{n_{r}} L_{2}
$$

ensuring that (1.6) holds.

To make the above arguments rigorous we need the following propositions.

Proposition 6.1. Let the elements of $\mathbf{L}$ be linearly independent over $\mathbb{Q}$. Let $1<v^{*}<v$ for $v \geqslant 3$. Given $\varepsilon>0$ there exist infinitely many $n \in \mathbb{N}$ such that

- for each $i=2, \ldots, v^{*}$ there exists $m \in \mathbb{N}$ satisfying

$$
\left|p_{m, i}-p_{n, 1}\right| \leqslant \varepsilon / 2
$$

- for all $i=v^{*}+1, \ldots, v$ and for all $m \in \mathbb{N}$

$$
\left|p_{m, i}-p_{n, 1}\right| \geqslant \frac{\pi}{2 L_{i}}-\varepsilon / 2 .
$$

Proof. The idea behind the proof is that for linearly independent elements of $\mathbf{L}$ the poles $p_{\cdot, i}$ for different $i$ behave like independent random variables, therefore every permitted pole configuration happens infinitely often. To substantiate this claim we express the nearest-pole distances as the states of an ergodic dynamical system.

For $n \in \mathbb{N}$ and $i=2, \ldots, v^{*}$, let $\delta_{n, i}$ denote the distance between $p_{n, 1}$ and the closest pole of $\tan k L_{i}$;

$$
\delta_{n, i}:=p_{n, 1}-p_{m, i}
$$

where $m$ is such that

$$
\left|p_{m, i}-p_{n, 1}\right|=\min _{m}\left\{\left|p_{m, i}-p_{n, 1}\right|\right\} .
$$

Since the poles of $\tan k L_{i}$ are $\pi / L_{i}$-periodic, we have

$$
\begin{aligned}
\delta_{n, i}+\frac{\pi}{2 L_{i}} & =p_{n, 1}-p_{0, i}+\frac{\pi}{2 L_{i}} \bmod \frac{\pi}{L_{i}} \\
& =\frac{\pi}{2 L_{1}}+\frac{\pi}{L_{1}} n \bmod \frac{\pi}{L_{i}} .
\end{aligned}
$$




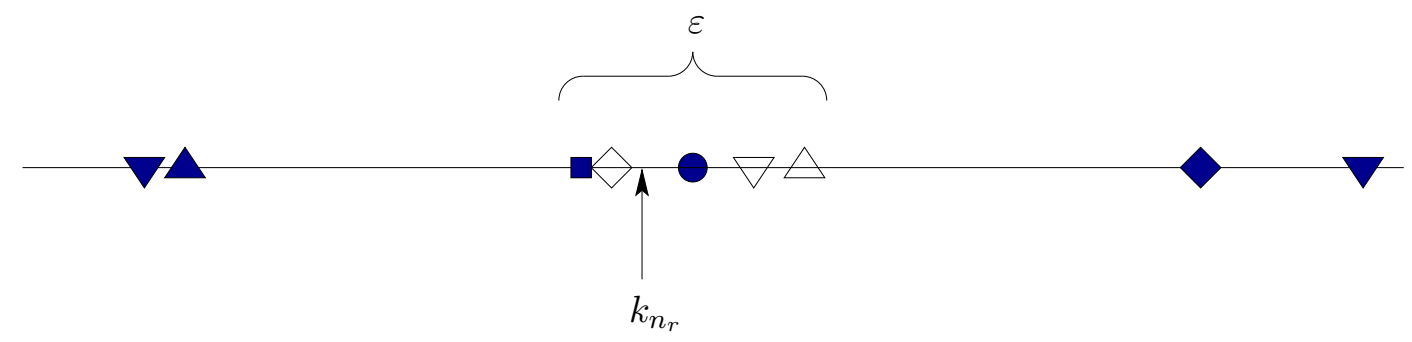

Figure 5: Poles $p_{n, i}$ and nodes $\ell_{n, i}$ on the real line. Different symbols correspond to different values of $i$ (the circle corresponds to $p_{n, 1}$ ). Filled symbols correspond to the poles, empty symbols to the nodes. In this example $v=5$ and $v^{*}=2$.

Let $\ell_{m, i}$ denote the $m^{\text {th }}$ zero of $\tan k L_{i}$. We note that (6.60) is implied by the condition that

$$
\left|\ell_{m, i}-p_{n, 1}\right| \leqslant \varepsilon / 2
$$

for some $m \in \mathbb{N}$. For $i=v^{*}+1, \ldots, v$, define $\eta_{n, i}$ to be the distance between $p_{n, 1}$ and the closest zero of $\tan k L_{i}$. Similarly to (6.62)

$$
\eta_{n, i}+\frac{\pi}{2 L_{i}}=\frac{\pi}{2}\left(\frac{1}{L_{1}}+\frac{1}{L_{i}}\right)+\frac{\pi}{L_{1}} n \bmod \frac{\pi}{L_{i}} .
$$

From (6.62) and (6.63),$\delta_{n, i}$ and $\eta_{n, i}$ satisfy the recurrence

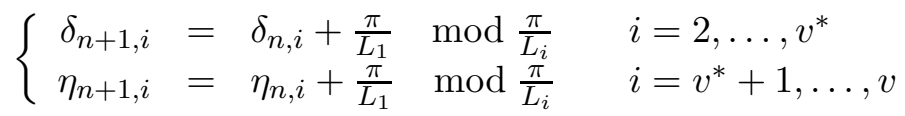

Since the bond lengths are not rationally related, the dynamical system (6.64) is equivalent to an irrational translation on a torus. In this case, Weyl's equidistribution result W applies, and any subset of the torus with positive Lebesgue measure is visited infinitely many times. The volume of the area in $\delta-\eta$ space defined by

$$
-\varepsilon / 2<\delta_{n, i}, \eta_{n, i}<\varepsilon / 2
$$

is non-zero and so there are infinitely many $n$ for which (6.65), and therefore (6.59,6.60), are satisfied.

The interpretation of proposition 6.1 is that we can find situations on the real line where $v^{*}$ poles of the functions $\tan k L_{i}$ are bunched together and the remaining $v-v^{*}$ poles are not close to these bunched poles (see figure 5).

Proposition 6.2. Under the conditions of proposition 6.1 there is a subsequence $\left(k_{n_{r}}\right) \subseteq$ $\left(k_{n}\right)$ for which

$$
\begin{aligned}
& \sec ^{2} k_{n_{r}} L_{i} \rightarrow \infty \quad \text { for } i=1, \ldots, v^{*} \\
& \sec ^{2} k_{n_{r}} L_{i} \rightarrow 1
\end{aligned}
$$

as $r \rightarrow \infty$. 
Proof. Let $\left(\varepsilon_{r}\right)$ be a sequence satisfying $\varepsilon_{r} \rightarrow 0$ as $r \rightarrow \infty$. We choose $k_{n_{r}}$ as follows.

Applying proposition 6.1 with $\varepsilon=\varepsilon_{r}$ yields a set of $v^{*}$ poles of $Z(k, \mathbf{L})$ inside a region with width $\varepsilon_{r}$. Since there is a zero of $Z(k, \mathbf{L})$ between any two poles of $Z(k, \mathbf{L})$, we can find $v^{*}-1$ zeros in this region. Set $k_{n_{r}}$ to be one of these zeros.

From proposition 6.1 we have

$$
\begin{aligned}
& \left|k_{n_{r}}-p_{m, i}\right| \leqslant \varepsilon_{r} \quad \text { for all } i=1, \ldots, v^{*} \text { and some } m=m(r, i) \\
& \left|k_{n_{r}}-\ell_{m, i}\right| \leqslant \varepsilon_{r} \quad \text { for all } i=v^{*}+1, \ldots, v \text { and some } m=m(r, i) .
\end{aligned}
$$

Since $\sec ^{2} L_{i} p_{m, i}=\infty, \sec ^{2} L_{i} \ell_{m, i}=1$ and $\sec \theta$ is a periodic function, the statement of the proposition follows trivially.

Corollary 6.3. If $v^{*}=2$ in proposition 6.2 then we additionally have

$$
\lim _{r \rightarrow \infty} \frac{\sec ^{2} k_{n_{r}} L_{1}}{\sec ^{2} k_{n_{r}} L_{2}}=1
$$

Proof. We recall that since $k_{n_{r}}$ is an eigenvalue, $Z\left(k_{n_{r}}, \mathbf{L}\right)=0$, and hence

$$
\tan k_{n_{r}} L_{1}=-\tan k_{n_{r}} L_{2}-\tan k_{n_{r}} L_{3}-\cdots-\tan k_{n_{r}} L_{v} .
$$

On the other hand, by proposition 6.2 $\tan k_{n_{r}} L_{i}$ remains bounded for $i>2$ and tends to infinity for $i=1,2$. Dividing (6.66) through by $\tan k_{n_{r}} L_{2}$ we obtain

$$
\lim _{r \rightarrow \infty} \frac{\tan k_{n_{r}} L_{1}}{\tan k_{n_{r}} L_{2}}=-1
$$

Further observations that $\sin ^{2} k_{n_{r}} L_{i} \rightarrow 1$ for $i=1,2$ and $\sec ^{2} \theta=\tan ^{2} \theta / \sin ^{2} \theta$ conclude the proof.

Lemma 6.4. Let $f:[0, L] \rightarrow \mathbb{R}$ be continuously differentiable. Then

$$
\lim _{k \rightarrow \infty} \int_{0}^{L} \cos (k x) f(x) \mathrm{d} x=0 .
$$

Proof. Integration by parts yields

$$
\int_{0}^{L} \cos (k x) f(x) \mathrm{d} x=\frac{1}{k}\left(\sin (k L) f(L)-\int_{0}^{L} \sin (k x) f^{\prime}(x) \mathrm{d} x\right)
$$

and the statement follows immediately from the boundedness of $f$ and its derivative.

Proof of theorem 1.4. Without loss of generality, we can assume that $i_{1}=1$ and $i_{2}=2$. We take the subsequence whose existence is guaranteed by proposition 6.2 with $v^{*}=2$. By corollary 6.3.

$$
\lim _{r \rightarrow \infty} A_{i}^{\left(n_{r}\right)^{2}}=2\left(\sum_{j=1}^{v} L_{j} \lim _{r \rightarrow \infty} \frac{\sec ^{2} k_{n_{r}} L_{j}}{\sec ^{2} k_{n_{r}} L_{i}}\right)^{-1}= \begin{cases}2\left(L_{1}+L_{2}\right)^{-1} & \text { if } i=1,2 \\ 0 & \text { otherwise }\end{cases}
$$

We use lemma 6.4 to get rid of the second integrals in (6.58) and conclude

$$
\lim _{r \rightarrow \infty}\left\langle\boldsymbol{\psi}^{\left(n_{r}\right)}|\mathbf{f}| \boldsymbol{\psi}^{\left(n_{r}\right)}\right\rangle=\frac{1}{L_{1}+L_{2}}\left(\int_{0}^{L_{1}} f_{1}(x) \mathrm{d} x+\int_{0}^{L_{2}} f_{2}(x) \mathrm{d} x\right) .
$$


Acknowledgements: GB is grateful to the University of Bristol for hospitality during visits while part of this research was carried out. BW wishes to thank the University of Strathclyde for hospitality.

BW has been financially supported by an EPSRC studentship (Award Number 0080052X).

We gratefully acknowledge the support of the European Commission under the Research Training Network (Mathematical Aspects of Quantum Chaos) HPRN-CT-200000103 of the IHP Programme.

\section{A Appendix: The order of integration in (4.45)}

In this appendix we deal with some technical issues regarding the exchange of order of integration in (4.45).

We first consider some asymptotics of $\tau_{\eta}(\xi)$.

Lemma A.1. For $\xi \in \mathbb{R}$,

$$
\tau_{\eta}(\xi)=\alpha|\xi|+\mathrm{O}_{\eta}\left(\xi^{-2}\right)
$$

as $|\xi| \rightarrow \infty$, where the error estimate depends on $\eta$.

Proof. We first note that $\tau_{\eta}$ is an even function, so we may assume $\xi>0$, and the result for $\xi<0$ will follow by symmetry. We can write $\tau_{\eta}(\xi)$ as

$$
\tau_{\eta}(\xi)=\sqrt{\eta t}\left(\frac{\xi}{\sqrt{\eta}}\right)+(\alpha-1) t(\xi)
$$

where

$$
t(\xi):=\frac{2}{\sqrt{\pi}} \exp \left(-\frac{\mathrm{i} \pi}{4}-\frac{\mathrm{i} \xi^{2}}{4}\right)+\xi \operatorname{erf}\left(\frac{\mathrm{e}^{\mathrm{i} \pi / 4} \xi}{2}\right) .
$$

We expand the error function asymptotically,

$$
\begin{aligned}
\operatorname{erf}\left(\frac{\mathrm{e}^{\mathrm{i} \pi / 4} \xi}{2}\right) & =1-\operatorname{erfc}\left(\frac{\mathrm{e}^{\mathrm{i} \pi / 4} \xi}{2}\right) \\
& =1-\frac{2}{\xi \sqrt{\pi}} \exp \left(-\frac{\mathrm{i} \xi^{2}}{4}-\frac{\mathrm{i} \pi}{4}\right)+\mathrm{O}\left(\xi^{-3}\right)
\end{aligned}
$$

as $\xi \rightarrow \infty$. Substituting (A.68) into A.67) gives

$$
t(\xi)=\xi+\mathrm{O}\left(\xi^{-2}\right), \quad \text { as } \xi \rightarrow \infty,
$$

and the lemma follows.

Lemma A.2. For $\xi>0, \mathfrak{R e} \frac{\mathrm{d} \tau_{\eta}}{\mathrm{d} \xi} \geqslant 0$ and for all $\xi \in \mathbb{R}$, there exists $\tau^{*}>0$ such that

$$
\mathfrak{R e} \tau_{\eta}(\xi) \geqslant \tau^{*} \text {. }
$$


Proof. By differentiation,

$$
\begin{aligned}
\frac{\mathrm{d} t}{\mathrm{~d} \xi} & =\operatorname{erf}\left(\frac{\mathrm{e}^{\mathrm{i} \pi / 4} \xi}{2}\right) \\
& =\frac{2}{\sqrt{\pi}} \mathrm{e}^{\mathrm{i} \pi / 4} \int_{0}^{\xi / 2} \mathrm{e}^{-\mathrm{i} r^{2}} \mathrm{~d} r .
\end{aligned}
$$

We see that

$$
\mathfrak{R e} \frac{\mathrm{d} t}{\mathrm{~d} \xi}=\frac{2}{\sqrt{\pi}} \int_{0}^{\xi / 2} \cos \left(r^{2}-\frac{\pi}{4}\right) \mathrm{d} r \geqslant 0
$$

Thus,

$$
\mathfrak{R e} \frac{\mathrm{d} \tau_{\eta}}{\mathrm{d} \xi}=\mathfrak{R e} t^{\prime}\left(\frac{\xi}{\sqrt{\eta}}\right)+(\alpha-1) \mathfrak{R e} t^{\prime}(\xi) \geqslant 0 .
$$

Hence it follows that $\mathfrak{R e} \tau_{\eta}(\xi) \geqslant \mathfrak{R e} \tau_{\eta}(0)=\sqrt{2}(\sqrt{\eta}+\alpha-1) / \sqrt{\pi}=: \tau^{*}$.

Lemma A.3. The integral

$$
\int_{0}^{\infty} P_{\eta}(\xi) \exp \left(-\sqrt{\beta} \tau_{\eta}(\xi)\right) \mathrm{d} \xi
$$

is uniformly convergent for $\beta \in\left[0, \beta_{0}\right]$ for all $\beta_{0}>0$.

Proof. By making the substitution $\nu=\xi^{2}$ we can consider the uniform convergence of

$$
\int^{\infty} P_{\eta}(\sqrt{\nu}) \exp \left(-\sqrt{\beta} \tau_{\eta}(\sqrt{\nu})\right) \frac{\mathrm{d} \nu}{\sqrt{\nu}}
$$

Let

$$
\begin{aligned}
f(\nu, \beta) & :=\frac{1}{\nu^{1 / 4}} \exp \left(-\sqrt{\beta} \mathfrak{R e} \tau_{\eta}(\sqrt{\nu})\right), \\
\phi(\nu, \beta) & :=\frac{P_{\eta}(\sqrt{\nu})}{\nu^{1 / 4}} \exp \left(-\sqrt{\beta} \mathrm{i} \mathfrak{I m} \tau_{\eta}(\sqrt{\nu})\right) .
\end{aligned}
$$

By lemma A.1 $\mathfrak{I m} \tau_{\eta}(\sqrt{\nu})=\mathrm{O}\left(\nu^{-1}\right)$ as $\nu \rightarrow \infty$ (We drop the $\eta$-dependence since we are concerned here only with fixed $\eta$ ). So

$$
\exp \left(-\sqrt{\beta} \mathrm{i} \mathfrak{I m} \tau_{\eta}(\sqrt{\nu})\right)=1+\mathrm{O}\left(\nu^{-1}\right)
$$

uniformly for $\beta \in\left[0, \beta_{0}\right]$. This means that

$$
\int^{\infty} \phi(\nu, \beta) \mathrm{d} \nu
$$

converges uniformly. i.e. given any $\varepsilon>0$ there exists $\nu_{1}$ such that for any $\nu_{2}>\nu_{1}$,

$$
\left|\int_{\nu_{1}}^{\nu_{2}} \phi(\nu, \beta) \mathrm{d} \nu\right|<\varepsilon
$$

for all $\beta \in\left[0, \beta_{0}\right] . f(\nu, \beta)$ is differentiable in $\nu$, and decreasing, so that

$$
\frac{\partial f}{\partial \nu} \leqslant 0
$$


If we let $\psi(\nu, \beta):=\int_{\nu_{1}}^{\nu} \phi\left(\nu^{\prime}, \beta\right) \mathrm{d} \nu^{\prime}$ then integrating by parts gives

$$
\begin{aligned}
\int_{\nu_{1}}^{\nu_{2}} f(\nu, \beta) \phi(\nu, \beta) \mathrm{d} \nu & =f\left(\nu_{2}, \beta\right) \psi\left(\nu_{2}, \beta\right)-\int_{\nu_{1}}^{\nu_{2}} \frac{\partial f}{\partial \nu}(\nu, \beta) \psi(\nu, \beta) \mathrm{d} \nu \\
& \leqslant f\left(\nu_{2}, \beta\right) \varepsilon-\varepsilon \int_{\nu_{1}}^{\nu_{2}} \frac{\partial f}{\partial \nu} \mathrm{d} \nu \\
& =\varepsilon f\left(\nu_{1}, \beta\right),
\end{aligned}
$$

where we have used the mean value theorem for integrals. If additionally, $\nu_{1}>1$ then $f\left(\nu_{1}, \beta\right)<1$ and we are done.

\section{Corollary A.4.}

$$
\int_{0}^{\infty} \int_{0}^{1} P_{\eta}(\xi) \mathrm{e}^{-\sqrt{\beta} \tau_{\eta}(\xi)-\mathrm{i} \sigma \beta} \mathrm{d} \beta \mathrm{d} \xi=\int_{0}^{1} \int_{0}^{\infty} P_{\eta}(\xi) \mathrm{e}^{-\sqrt{\beta} \tau_{\eta}(\xi)-\mathrm{i} \sigma \beta} \mathrm{d} \xi \mathrm{d} \beta
$$

Proof. This follows immediately from lemma A.3, see, for example, $\S 11.55$.II of [St].

Lemma A.5. The integral

$$
\int_{1}^{\infty} \exp \left(-\sqrt{\beta} \tau_{\eta}(\xi)-\mathrm{i} \sigma \beta\right) \mathrm{d} \beta
$$

is uniformly convergent for $\xi \in\left[0, \xi_{0}\right]$ for all $\xi_{0}>0$.

Proof. We, in fact, prove the stronger statement that the integral in question is uniformly convergent for all $\xi>0$. Taking $M(\beta):=\mathrm{e}^{-\tau^{*} \sqrt{\beta}}$,

$$
\left|\exp \left(-\sqrt{\beta} \tau_{\eta}(\xi)-\mathrm{i} \sigma \beta\right)\right| \leqslant M(\beta)
$$

and the integral is uniformly convergent by the Weierstrass $M$-test.

Lemma A.6. The iterated integral

$$
\int_{0}^{\infty} \int_{1}^{R^{2}} P_{\eta}(\xi) \mathrm{e}^{-\sqrt{\beta} \tau_{\eta}(\xi)-\mathrm{i} \sigma \beta} \mathrm{d} \beta \mathrm{d} \xi
$$

converges uniformly for $R>1$.

Proof. We shall first consider the case where $\sigma<0$. A lengthy calculation gives

$$
\begin{aligned}
\int_{1}^{R^{2}} \mathrm{e}^{-\sqrt{\beta} \tau_{\eta}(\xi)-\mathrm{i} \sigma \beta} \mathrm{d} \beta= & \frac{1}{\mathrm{i} \sigma} \mathrm{e}^{-\mathrm{i} \sigma-\tau_{\eta}(\xi)}-\frac{1}{\mathrm{i} \sigma} \mathrm{e}^{-\mathrm{i} R^{2} \sigma-R \tau_{\eta}(\xi)} \\
& -\frac{\sqrt{\pi} \tau_{\eta}(\xi)}{2 \mathrm{e}^{-3 \pi \mathrm{i} / 4}(-\sigma)^{3 / 2}} \exp \left(\frac{\tau_{\eta}(\xi)^{2}}{4 \mathrm{i} \sigma}\right)\left[\operatorname{erfc}\left(\mathrm{e}^{-\mathrm{i} \pi / 4} \sqrt{-\sigma}+\frac{\tau_{\eta}(\xi) \mathrm{e}^{\mathrm{i} \pi / 4}}{2 \sqrt{-\sigma}}\right)\right. \\
& \left.-\operatorname{erfc}\left(R \mathrm{e}^{-\mathrm{i} \pi / 4} \sqrt{-\sigma}+\frac{\tau_{\eta}(\xi) \mathrm{e}^{\mathrm{i} \pi / 4}}{2 \sqrt{-\sigma}}\right)\right] .
\end{aligned}
$$

By Lemma A.1 $\tau_{\eta}(\xi) \sim \alpha \xi$ as $\xi \rightarrow \infty$, so

$$
\int_{0}^{\infty} P_{\eta}(\xi) \mathrm{e}^{-\mathrm{i} R^{2} \sigma-R \tau_{\eta}(\xi)} \mathrm{d} \xi
$$


is uniformly convergent for $R>1$ by the Wierstrass $M$-test with $M(\xi):=C \mathrm{e}^{-\tau_{\eta}(\xi)}$ for some constant $C$ which does not depend on $\xi$.

We can write

$$
\begin{aligned}
\exp \left(\frac{-\mathrm{i} \tau_{\eta}(\xi)^{2}}{4 \sigma}\right) & \operatorname{erfc}\left(R \mathrm{e}^{-\mathrm{i} \pi / 4} \sqrt{-\sigma}+\frac{\tau_{\eta}(\xi) \mathrm{e}^{\mathrm{i} \pi / 4}}{2 \sqrt{-\sigma}}\right) \\
& =\exp \left(-R^{2} \mathrm{i} \sigma-R \tau_{\eta}(\xi)\right) w\left(R \mathrm{e}^{\mathrm{i} \pi / 4} \sqrt{-\sigma}+\frac{\tau_{\eta}(\xi) \mathrm{e}^{3 \pi \mathrm{i} / 4}}{2 \sqrt{-\sigma}}\right)
\end{aligned}
$$

and since $w(z)=\mathrm{O}\left(z^{-1}\right)$ as $z \rightarrow \infty$ and $\left|\tau_{\eta}(\xi)\right| \geqslant \tau^{*}$,

$$
w\left(R \mathrm{e}^{\mathrm{i} \pi / 4} \sqrt{-\sigma}+\frac{\tau_{\eta}(\xi) \mathrm{e}^{3 \pi \mathrm{i} / 4}}{2 \sqrt{-\sigma}}\right)=\mathrm{O}(1)
$$

as $\xi \rightarrow \infty$, uniformly for $R>1$. Since $\left|\exp \left(-R \tau_{\eta}(\xi)\right)\right| \leqslant \exp \left(-\tau_{\eta}(\xi)\right)$ we see that the convergence of

$$
\int_{0}^{\infty} P_{\eta}(\xi) \tau_{\eta}(\xi) \exp \left(\frac{-\mathrm{i} \tau_{\eta}(\xi)^{2}}{4 \sigma}\right) \operatorname{erfc}\left(R \mathrm{e}^{-\mathrm{i} \pi / 4} \sqrt{-\sigma}+\frac{\tau_{\eta}(\xi) \mathrm{e}^{\mathrm{i} \pi / 4}}{2 \sqrt{-\sigma}}\right) \mathrm{d} \xi
$$

is uniform for $R>1$, by the Wierstrass $M$-test.

In the case $\sigma=0$ we have the simpler integral

$$
\begin{aligned}
\int_{1}^{R^{2}} \exp \left(-\sqrt{\beta} \tau_{\eta}(\xi)\right) \mathrm{d} \beta= & \frac{2}{\tau_{\eta}(\xi)^{2}}\left(\tau_{\eta}(\xi)\left(\mathrm{e}^{-\tau_{\eta}(\xi)}-R \mathrm{e}^{-R \tau_{\eta}(\xi)}\right)\right. \\
& \left.+\left(\mathrm{e}^{-\tau_{\eta}(\xi)}-\mathrm{e}^{-R \tau_{\eta}(\xi)}\right)\right) .
\end{aligned}
$$

The integral with respect to $\xi$ then converges uniformly by the Wierstrass $M$-test, since

$$
\left|\exp \left(-R \tau_{\eta}(\xi)\right)\right| \leqslant \exp \left(-\mathfrak{R e} \tau_{\eta}(\xi)\right)
$$

and

$$
\left|R \exp \left(-R \tau_{\eta}(\xi)\right)\right| \leqslant \frac{2}{\tau^{*} \mathrm{e}} \exp \left(-\frac{1}{2} \mathfrak{R e} \tau_{\eta}(\xi)\right)
$$

for $R>1$.

The following theorem from $\S 11.55$.III of [St] describes criteria which permit the interchange of order of two improper integrals.

Theorem A.7. Let $f(x, \alpha)$ be continuous in $\alpha_{1} \leqslant \alpha \leqslant \alpha_{2}$ and $c \leqslant x \leqslant d$, where both $\alpha_{2}$ and $d$ may be arbitrarily large, and;

$$
\begin{aligned}
& \text { i) } \int_{c}^{\infty} f(x, \alpha) \mathrm{d} x \quad \text { be uniformly convergent for } \alpha \in\left[\alpha_{1}, \alpha_{2}\right] \text {, } \\
& \text { ii) } \int_{\alpha_{1}}^{\infty} f(x, \alpha) \mathrm{d} \alpha \quad \text { be uniformly convergent for } x \in[c, d] \text {, } \\
& \text { iii) } \int_{c}^{\infty} \int_{\alpha_{1}}^{R} f(x, \alpha) \mathrm{d} \alpha \mathrm{d} x \text { be uniformly convergent for } R \in\left[\alpha_{1}, \infty\right] \text {. }
\end{aligned}
$$

then

$$
\int_{\alpha_{1}}^{\infty} \int_{c}^{\infty} f(x, \alpha) \mathrm{d} x \mathrm{~d} \alpha=\int_{c}^{\infty} \int_{\alpha_{1}}^{\infty} f(x, \alpha) \mathrm{d} \alpha \mathrm{d} x
$$


Applying theorem A.7 to the integral in (4.45) allows us to conclude the following.

\section{Proposition A.8.}

$$
\int_{0}^{\infty} \int_{0}^{\infty} P_{\eta}(\xi) \exp \left(-\sqrt{\beta} \tau_{\eta}(\xi)-\mathrm{i} \sigma \beta\right) \mathrm{d} \xi \mathrm{d} \beta=\int_{0}^{\infty} \int_{0}^{\infty} P_{\eta}(\xi) \exp \left(-\sqrt{\beta} \tau_{\eta}(\xi)-\mathrm{i} \sigma \beta\right) \mathrm{d} \beta \mathrm{d} \xi .
$$

Proof. This follows from theorem A.7 with lemmas A.3. A.5 and A.6 together with corollary A.4.

\section{B Appendix: Simplification of (5.55)}

We here consider some technical points that arise in section 5

Lemma B.1. The integral

$$
\mathfrak{R e} \int_{0}^{\infty} P_{\eta}(\xi)\left(\frac{\mathrm{e}^{3 \mathrm{i} \pi / 4} \tau_{\eta}(\xi)}{2(-\sigma)^{3 / 2}} w\left(\frac{\mathrm{e}^{3 \mathrm{i} \pi / 4} \tau_{\eta}(\xi)}{2 \sqrt{-\sigma}}\right)\right) \mathrm{d} \xi
$$

is uniformly convergent for $\sigma \in\left[-R^{2}, 0\right]$ for any $R>0$.

Proof. Expanding the $w$ function, using lemma 4.2

$$
\frac{\mathrm{e}^{3 \pi \mathrm{i} / 4} \tau_{\eta}(\xi)}{2(-\sigma)^{3 / 2}} w\left(\frac{\mathrm{e}^{3 \mathrm{i} \pi / 4} \tau_{\eta}(\xi)}{2 \sqrt{-\sigma}}\right)=\frac{1}{\mathrm{i} \sigma}+\mathrm{O}\left(\frac{1}{\tau_{\eta}(\xi)^{2}}\right)
$$

as $\xi \rightarrow \infty$ where the implied constant is independent of $\sigma \in[-R, 0]$. Since $\int_{0}^{\infty} P_{\eta}(\xi) \mathrm{d} \xi=\alpha$ the leading order term in the expansion of (B.73) has zero real part, and the integral of the remainder converges since $\tau_{\eta}(\xi)^{-2} \sim(\alpha \xi)^{-2}$ as $\xi \rightarrow \infty$.

Proposition B.2. We have

$$
\lim _{R \rightarrow \infty} \int_{0}^{\infty} \int_{-\infty}^{-R^{2}} P_{\eta}(\xi)\left(\frac{\mathrm{e}^{3 \mathrm{i} \pi / 4} \tau_{\eta}(\xi)}{2(-\sigma)^{3 / 2}} w\left(\frac{\mathrm{e}^{3 \mathrm{i} \pi / 4} \tau_{\eta}(\xi)}{2 \sqrt{-\sigma}}\right)\right) \mathrm{d} \sigma \mathrm{d} \xi=0
$$

Proof. We make the substitution $2 p=(-\sigma)^{-1 / 2}$ to give

$$
\begin{aligned}
\int_{-\infty}^{-R^{2}} \frac{\mathrm{e}^{3 \mathrm{i} \pi / 4} \tau_{\eta}(\xi)}{2(-\sigma)^{3 / 2}} w\left(\frac{\mathrm{e}^{3 \mathrm{i} \pi / 4} \tau_{\eta}(\xi)}{2 \sqrt{-\sigma}}\right) \mathrm{d} \sigma & =\int_{0}^{1 / 2 R} 2 \mathrm{e}^{3 \mathrm{i} \pi / 4} \tau_{\eta}(\xi) w\left(\mathrm{e}^{3 \mathrm{i} \pi / 4} \tau_{\eta}(\xi) p\right) \mathrm{d} p \\
& =\int_{\gamma_{\xi, R}} 2 w(t) \mathrm{d} t
\end{aligned}
$$

where $t \in \mathbb{C}$ follows the contour $\gamma_{\xi, R}$ connecting 0 to $\frac{\mathrm{e}^{3 \mathrm{i} \pi / 4} \tau_{\eta}(\xi)}{2 R}$. Since $w$ is an analytic function, we can write

$$
\int_{-\infty}^{-R^{2}} \frac{\mathrm{e}^{3 \mathrm{i} \pi / 4} \tau_{\eta}(\xi)}{2(-\sigma)^{3 / 2}} w\left(\frac{\mathrm{e}^{3 \mathrm{i} \pi / 4} \tau_{\eta}(\xi)}{2 \sqrt{-\sigma}}\right) \mathrm{d} \sigma=2 W\left(\frac{\mathrm{e}^{3 \mathrm{i} \pi / 4} \tau_{\eta}(\xi)}{2 R}\right)
$$


where $W$ is the antiderivative of $w$ satisfying

$$
\frac{\mathrm{d} W}{\mathrm{~d} z}=w(z) \quad \text { and } \quad W(0)=0 .
$$

By making the substitution $\xi \mapsto R \xi$, we see that

$$
\begin{aligned}
\int_{0}^{\infty} P_{\eta}(\xi) W\left(\frac{\mathrm{e}^{3 \mathrm{i} \pi / 4} \tau_{\eta}(\xi)}{2 R}\right) \mathrm{d} \nu= & \frac{R}{2} \int_{0}^{1} P_{\eta}(R \sqrt{\nu}) W\left(\frac{\mathrm{e}^{3 \mathrm{i} \pi / 4} \tau_{\eta}(R \sqrt{\nu})}{2 R}\right) \frac{\mathrm{d} \nu}{\sqrt{\nu}} \\
& +R \int_{1}^{\infty} P_{\eta}(R \xi) W\left(\frac{\mathrm{e}^{3 \mathrm{i} \pi / 4} \tau_{\eta}(R \xi)}{2 R}\right) \mathrm{d} \xi
\end{aligned}
$$

where we have, additionally, split the range of integration into two regimes and the first integral made the substitution $\nu=\xi^{2}$. For the first integral in (B.74) we consider

$$
\int_{0}^{1} \frac{R}{2} \exp \left(\frac{\mathrm{i} \pi}{4}-\frac{\mathrm{i} R^{2} \nu}{4}\right) W\left(\frac{\mathrm{e}^{3 \mathrm{i} \pi / 4} \tau_{\eta}(R \sqrt{\nu})}{2 R}\right) \frac{\mathrm{d} \nu}{\sqrt{\nu}}
$$

which comes from the first term of $P_{\eta}(R \sqrt{\nu})$. The second term of $P_{\eta}$ can be handled in the same way. Differentiating,

$$
\frac{\mathrm{d}}{\mathrm{d} \nu}\left[W\left(\frac{\mathrm{e}^{3 \mathrm{i} \pi / 4} \tau_{\eta}(R \sqrt{\nu})}{2 R}\right)\right]=\frac{\mathrm{e}^{3 \mathrm{i} \pi / 4}}{4 \sqrt{\nu}} w\left(\frac{\mathrm{e}^{3 \mathrm{i} \pi / 4} \tau_{\eta}(R \sqrt{\nu})}{2 R}\right) \tau_{\eta}^{\prime}(R \sqrt{\nu}) .
$$

Since $\frac{\mathrm{d} \tau_{\eta}}{\mathrm{d} \xi}$ is bounded for $\xi \in \mathbb{R}$, we deduce from $(\underline{B .75})$ that there exists a constant $K$ independent of $R$ such that

$$
\left|\frac{\mathrm{d}}{\mathrm{d} \nu}\left[W\left(\frac{\mathrm{e}^{3 \mathrm{i} \pi / 4} \tau_{\eta}(R \sqrt{\nu})}{2 R}\right)\right]\right| \leqslant \frac{K}{\sqrt{\nu}} .
$$

Let

$$
\psi(\nu):=-\sqrt{\pi} \operatorname{erfc}\left(\frac{R \mathrm{e}^{\mathrm{i} \pi / 4} \sqrt{\nu}}{2}\right)
$$

which satisfies

$$
\frac{\mathrm{d} \psi}{\mathrm{d} \nu}=\frac{R}{2 \sqrt{\nu}} \exp \left(\frac{\mathrm{i} \pi}{4}-\frac{\mathrm{i} R^{2} \nu}{4}\right) .
$$

We can then use integration by parts,

$$
\begin{aligned}
\int_{0}^{1} \frac{R}{2} & \exp \left(\frac{\mathrm{i} \pi}{4}-\frac{\mathrm{i} R^{2} \nu}{4}\right) W\left(\frac{\mathrm{e}^{3 \mathrm{i} \pi / 4} \tau_{\eta}(R \sqrt{\nu})}{2 R}\right) \frac{\mathrm{d} \nu}{\sqrt{\nu}} \\
= & {\left[\psi(\nu) W\left(\frac{\mathrm{e}^{3 \mathrm{i} \pi / 4} \tau_{\eta}(R \sqrt{\nu})}{2 R}\right)\right]_{0}^{1}-\int_{0}^{1} \psi(\nu) \frac{\mathrm{d}}{\mathrm{d} \nu}\left[W\left(\frac{\mathrm{e}^{3 \mathrm{i} \pi / 4} \tau_{\eta}(R \sqrt{\nu})}{2 R}\right)\right] \mathrm{d} \nu } \\
& \rightarrow 0
\end{aligned}
$$

as $R \rightarrow \infty$, since

$$
W\left(\frac{\mathrm{e}^{3 \mathrm{i} \pi / 4} \tau_{\eta}(0)}{2 R}\right) \rightarrow 0
$$


and

$$
\operatorname{erfc}\left(\frac{R \mathrm{e}^{\mathrm{i} \pi / 4}}{2}\right) \rightarrow 0
$$

and the fact that the final integral in (B.78) converges uniformly by (B.76).

For the second integral in (B.74) we apply Taylor's theorem and lemma A.1 to get

$$
W\left(\frac{\mathrm{e}^{3 \mathrm{i} \pi / 4} \tau_{\eta}(R \xi)}{2 R}\right)=W\left(\frac{\mathrm{e}^{3 \mathrm{i} \pi / 4} \alpha \xi}{2}\right)+\mathrm{O}\left(\frac{1}{R^{3} \xi^{2}}\right)
$$

This gives

$$
R \int_{1}^{\infty} P_{\eta}(R \xi) W\left(\frac{\mathrm{e}^{3 \mathrm{i} \pi / 4} \tau_{\eta}(R \xi)}{2 R}\right) \mathrm{d} \xi=R \int_{1}^{\infty} P_{\eta}(R \xi) W\left(\frac{\mathrm{e}^{3 \mathrm{i} \pi / 4} \alpha \xi}{2}\right) \mathrm{d} \xi+\mathrm{O}\left(R^{-2}\right)
$$

as $R \rightarrow \infty$. The integral which remains is of a form for which the asymptotic series may be derived by the method of repeated integration-by-parts $\mathrm{BlHa}$ to see that this contribution also vanishes in the limit $R \rightarrow \infty$.

\section{References}

[AS] Abramowitz M and Stegun I A 1965 Handbook of mathematical functions (Dover)

[BSS] Bäcker A, Schubert R and Sifter P 1998 Rate of quantum ergodicity in Euclidean billiards Phys. Rev. E 57 5425-5447, erratum ibid. 585192

[BG] Barra F and Gaspard P 2000 On the level spacing distribution in quantum graphs J. Stat. Phys. 101 283-319

[B] Berkolaiko G Form factor for large quantum graphs: evaluating orbits with time-reversal preprint available at arXiv:nlin.CD/0305009

[BBK] Berkolaiko G, Bogomolny E B and Keating J P 2001 Star graphs and Šeba billiards J. Phys. A $\mathbf{3 4} 335-350$

[BK] Berkolaiko G and Keating J P 1999 Two-point spectral correlations for star graphs J. Phys. A 32 7827-7841

[BSW1] Berkolaiko G, Schanz H and Whitney R S 2002 Leading off-diagonal correction to the form factor of large graphs Phys. Rev. Lett. 82 art. no. 104101

[BSW2] Berkolaiko G, Schanz H and Whitney R S 2003 Form factor for a family of quantum graphs: An expansion to third order J. Phys. A 36 8373-8392

[BKW] Berkolaiko G, Keating J P and Winn B Intermediate wave-function statistics preprint available at arXiv:nlin.CD/0304034

[Be1] Berry M V 1977 Regular and irregular semiclassical wavefunctions J. Phys. A $102083-2091$ 
[Be2] Berry M V 1989 Quantum scars of classical closed orbits in phase space Proc. Roy. Soc. Lond. A 423 219-231

[BlHa] Bleistein N and Handlesman R A 1986 Asymptotic expansions of integrals (Dover)

[Bo] Bogomolny E B 1988 Smoothed wave functions of chaotic quantum systems Physica D 31 169-189

[BH1] Bolte J and Harrison J 2003 Spectral statistics for the Dirac operator on graphs J. Phys. A 36 2747-2769

[BH2] Bolte $\mathrm{J}$ and Harrison J 2003 The spin contribution to the form factor of quantum graphs J. Phys. A 36 L433-L440

[CdV] Colin de Verdière Y 1985 Ergodicité et fonctions propres du Laplacien Commun. Math. Phys 102 497-502

[CDM] Comtet A, Desbois J and Majumdar S N 2002 The local time distribution of a particle diffusing on a graph $J$. Phys. A 35 687-694

[D] Desbois J 2002 Occupation times distribution for Brownian motion on graphs J. Phys. A 35 673-678

[DS] Dunford N and Schwartz J T 1963 Linear Operators Part II: Spectral Theory (Interscience Publishers)

[FNdB] Faure F, Nonnenmacher S and de Bièvre S Scarred eigenstates for quantum cat maps of minimal periods preprint available at arXiv:nlin.CD/0207060

[GL] Gérard P and Leichtnam E 1993 Ergodic properties of the eigenfunctions for the Dirichlet problem Duke Math. J. 71 559-607

[GR] Gradshteyn I S and Ryzhik I M 1965 Tables of integrals, series, and products (Academic Press)

[GSW] Gnutzmann S, Smilansky U and Weber J Nodal domains on quantum graphs preprint available at arXiv:nlin.CD/0305020

[H] Heller E J 1984 Bound-state eigenfunctions of classically chaotic Hamiltonian systems: scars of periodic orbits Phys. Rev. Lett. 53 1515-1518

[K1] Kaplan L 1999 Scars in quantum chaotic wavefunctions Nonlinearity 12 R1-R40

[K2] Kaplan L 2001 Eigenstate structure in graphs and disordered lattices Phys. Rev. E 64 art. no. 036225

[KH] Kaplan L and Heller E J 1998 Linear and nonlinear theory of eigenfunction scars Ann. Phys. 264 171-206

[Ke] Keating J P 1991 The cat maps: quantum mechanics and classical motion Nonlinearity 4 309-341 
[KMW] Keating J P, Marklof J and Winn B Value distribution of the eigenfunctions and spectral determinants of quantum star graphs. To appear in Commun. Math. Phys. Preprint available at arXiv:math-ph/0210060

[KP] Keating J P and Prado S D 2001 Orbit bifurcations and the scarring of wavefunctions Proc. Roy. Soc. Lond. A 457 1855-1872

[KS1] Kottos T and Smilansky U 1997 Quantum Chaos on graphs Phys. Rev. Lett. $794794-4797$

[KS2] Kottos T and Smilansky U 1999 Periodic orbit theory and spectral statistics for quantum graphs Ann. Phys. 274 76-124

[KS3] Kottos T and Smilansky U 2000 Chaotic scattering on graphs Phys. Rev. Lett. $85968-971$

[KS4] Kottos T and Smilansky U 2003 Quantum graphs: a simple model for chaotic scattering J. Phys. A 36 3501-3524

[PTZ] Pakoński P, Tanner G and Życzkowski K 2003 Families of line-graphs and their quantization J. Stat. Phys. 111 1331-1351

[S] Schnirelmann A 1974 Ergodic properties of eigenfuncions Usp. Math. Nauk. 29 $181-182$

[St] Stewart C A 1940 Advanced Calculus (Methuen)

[Se] Šeba P 1990 Wave chaos in singular quantum billiards Phys. Rev. Lett. 64 $1855-1858$

[SK] Schanz H and Kottos T 2003 Scars on quantum networks ignore the Lyapunov exponent Phys. Rev. Lett. 90 art. no. 234101

[T] Tanner G 2001 Unitary stochastic matrix ensembles and spectral statistics $J$. Phys. A 34 369-383

[TM] Texier C and Montambaux G 2001 Scattering theory on graphs J. Phys. A 34 $10307-10326$

[V] Voros A 1979 Semi-classical ergodicity of quantum eigenstates in the Wigner representation, in Stochastic behaviour in classical and quantum Hamiltonian systems (Springer-Verlag) pp. 326-333

[W] Weyl H 1916 Über die Gleichverteilung von Zahlen mod. Eins Math. Ann. 77 313-352

[Z] Zelditch S 1987 Uniform distribution of the eigenfunctions on compact hyperbolic surfaces Duke Math. J. 55 919-941 